\title{
BMJ Open Comparison of the safety and efficacy between linear stapler and circular stapler in totally laparoscopic total gastrectomy: protocol for a systematic review and meta-analysis
}

Tianyou Liao, ${ }^{1}$ Leilei Deng, ${ }^{2}$ Xueqing Yao, ${ }^{3,4}$ Manzhao Ouyang ${ }^{1,3}$

To cite: Liao T, Deng L, Yao X, et al. Comparison of the safety and efficacy between linear stapler and circular stapler in totally laparoscopic total gastrectomy: protocol for a systematic review and meta-analysis. BMJ Open 2019;9:e028216. doi:10.1136/ bmjopen-2018-028216

- Prepublication history for this paper is available online. To view these files, please visit the journal online (http://dx.doi org/10.1136/bmjopen-2018028216).

$T L$ and $L D$ contributed equally. $\mathrm{XY}$ and $\mathrm{MO}$ contributed equally.

Received 27 November 2018 Revised 4 April 2019 Accepted 4 April 2019

A Check for updates

(C) Author(s) (or their employer(s)) 2019. Re-use permitted under CC BY-NC. No commercial re-use. See rights and permissions. Published by BMJ.

For numbered affiliations see end of article.

\section{Correspondence to}

Dr Manzhao Ouyang;

ouyangmanzhao@163.com and

Professor Xueqing Yao;

syyaoxueqing@scut.edu.cn

\section{ABSTRACT}

Introduction Total gastrectomy is often recommended for upper body gastric cancer, and totally laparoscopic total gastrectomy (TLTG) is deemed to be a promising surgical method with the well-known advantages such as less invasion and fast recovery. However, the anastomosis between oesophagus and jejunum is the difficulty of TLTG. Although staplers have promoted the development of TLTG, the choice of suitable staplers to complete oesophagojejunostomy is controversial and unclear. Therefore, a higher level of research evidence is needed to compare the two types of staplers in terms of safety and efficacy for oesophagojejunostomy in TLTG among patients with gastric cancer.

Methods and analysis PubMed, Embase, Cochrane Library, China National Knowledge Infrastructure (CNKI) and Wanfang Databases will be comprehensively searched from January 1990 to July 2019. All eligible randomised controlled trials (RCTs), non-RCTs or observational studies comparing the two types of staplers will be included. A meta-analysis will be performed using Review Manager V.5.3 software to compare the safety and efficacy of linear and circular staplers for oesophagojejunostomy in TLTG. The primary outcomes are anastomotic leakage, anastomotic stricture, anastomotic haemorrhage. The secondary outcomes include time to first instance of passing gas after surgery, first feeding time, total operation time, reconstruction time, estimated blood loss. The heterogeneity of this study will be assessed by $p$ values and $\mathrm{I}^{2}$ statistic. Subgroup analyses and sensitivity analyses will be used to explore and explain the heterogeneity. The risk of bias will be assessed using the Cochrane tool or the NewcastleOttawa Quality Assessment Scale.

Ethics and dissemination Ethical approval will not be required because this proposed systematic review and metaanalysis is based on previously published data, which does not include intervention data on patients. The findings of this study will be submitted to a peer-reviewed journal and will be presented at a relevant congress.

PROSPERO registration number CRD42018111680.

\section{INTRODUCTION}

Gastric cancer is a common malignant tumour of the digestive tract, and its morbidity and mortality ranked fifth and third, respectively,
Strengths and limitations of this study

- To our best knowledge, this review will be the first systematic review and meta-analysis to compare the safety and efficacy of the linear stapler and circular staplers in totally laparoscopic total gastrectomy.

- The study selection, data extraction and quality assessment of the studies will be performed by three independent reviewers.

- Subgroup analyses and sensitivity analyses will be used to explore and explain the heterogeneity.

- Some observational studies might be included in this study, which might affect the quality of the data.

- There might be some selection bias in this systematic review and meta-analysis, because the resource databases are limited to English and Chinese language.

among the global malignant tumours. Although the overall incidence of gastric cancer has been decreasing worldwide, the incidence of upper body gastric cancer has been on an increasing trend. ${ }^{23}$ Radical resection is the only curative modality recommended for primary treatment of patients with resectable gastric cancer, and total gastrectomy is often performed for upper body gastric cancer. ${ }^{3} 4$ Laparoscopic technique is one of the main development direction of surgical treatment for gastric cancer. The results of a multicentre retrospective cohort study have shown that laparoscopic total gastrectomy (LTG) could achieve comparable oncological outcomes to open total gastrectomy. ${ }^{5}$ Furthermore, with the development of new laparoscopic equipments and the accumulation of advanced experience in the application of laparoscopic techniques, laparoscopic surgery for gastric cancer has undergone a technological transition from laparoscopic-assisted surgery to 
totally laparoscopic surgery which is less invasive and expedites postoperative recovery. ${ }^{6}$

However, the anastomosis and reconstruction of oesophagojejunostomy is the focal point and difficulty of totally laparoscopic total gastrectomy (TLTG). ${ }^{6}$ Presently, the two commonly used anastomosis methods for oesophagojejunostomy are circular stapler anastomosis and linear stapler anastomosis. ${ }^{6-8}$ Considering the characteristics of laparoscopic surgery, traditional circular anastomosis has certain inherent limitations. For example, the circular stapler cannot be placed through a trocar, and it needs to be placed in the abdominal cavity through a small assisted incision in the abdomen, thereby reducing the benefit of laparoscopic surgery. Although OrVil does not pass through the abdominal cavity, a top-down placement method is required, but the operation requires an anaesthesiologist to cooperate. ${ }^{9}$ Compared with the circular stapler, linear stapler has some advantages in oesophagojejunostomy. ${ }^{10}$ For example, it can easily enter the abdominal cavity through the trocar, without purse-string suture, and the used instrument is easy to operate. The primary disadvantage of linear anastomosis is the need for a longenough oesophageal stump for anastomosis, which limits the surgical margin and could increase the tension of the anastomosis. For this reason, some scholars consider that linear anastomosis is not appropriate for patients with tumours located in the upper stomach or close to the oesophagogastric junction or tumours with oesophageal invasion. ${ }^{11} 12$ A meta-analyses comparing linear anastomosis with circular anastomosis in laparoscopic distal gastrectomy (LDG) suggested that linear anastomosis is better than circular anastomosis in LDG. ${ }^{13}$ However, considering the differences between TLTG and LDG in terms of surgical methods, surgical objects and surgical difficulties, this conclusion cannot be applied to guide the implementation of TLTG.

Therefore, the choice of staplers for complete oesophagojejunostomy of TLTG is still an unclear and controversial topic. ${ }^{7814}$ Majority of the previous comparisons on contrasting linear and circular stapling anastomosis for oesophagojejunostomy in TLTG are retrospective and are based on small-sample studies, further, the results from such investigation have been inconsistent and even contradictory. Therefore, the safety and efficacy of linear stapling anastomosis has not been well resolved in these studies and remains to be confirmed by higher-level evidence. In view of this, a systematic review and meta-analysis will be conducted based on relevant published literature to further explore and compare the safety and efficacy of the linear stapler and circular stapler in TLTG, with the hope of providing a reference to help surgeons choose a suitable stapler.

\section{MATERIALS AND METHODS}

The protocol of the planned systematic review and meta-analysis was prepared in accordance with the recommendation from the Preferred Reporting Items for
Systematic Review and Meta-Analysis (PRISMA) Protocols statement, ${ }^{15}$ and this systematic review and meta-analysis will be written in line with PRISMA statement. ${ }^{16}$ In addition, this study protocol was registered with the international prospective register of systematic reviews PROSPERO. ${ }^{17}$

\section{Literature-search strategy}

Relevant studies will be searched on PubMed, Cochrane Library, Embase, CNKI and Wanfang Databases in accordance with the population, intervention, control and outcomes criteria from January 1990 to July 2019. The studies comparing linear stapler with circular stapler for oesophagojejunostomy in TLTG will be included. The following MeSH terms and their combinations will be searched in (Title/Abstract): (1) 'linear stapler' OR 'overlap' OR 'FEEA' OR 'functional end-to-end anastomosis' OR 'T-shaped' OR ' $\varpi$-shaped' OR 'delta-shaped'; (2) 'circular stapler' OR 'OrVil ${ }^{T M}$, OR 'hemidouble stapling technique' OR 'double stapling technique'; (3) 'totally laparoscopic'; (4) 'total gastrectomy'. The related-articles function will be used to increase the search scope, and the computer search will be supplemented with manual screening of the reference lists of all retrieved studies, review articles and conference abstracts.

\section{Inclusion criteria}

(1) The subjects were the patients who had undergone oesophagojejunostomy in TLTG, and preoperative or postoperative histopathological examination confirmed gastric cancer; (2) according to the different anastomosis methods used for oesophagojejunostomy in digestive tract reconstruction, patients were divided into linear stapling anastomosis and circular stapling anastomosis groups; (3) the study types were randomised controlled trials (RCTs), non-RCTs or observational comparative studies; (4) the original literature should have terms including intraoperative conditions, postoperative specimens, postoperative recovery, postoperative complications, postoperative complications or have at least one of these research data; (5) pooled results can be formulated by the statistical index, such as OR, relative risk or weighted mean difference (WMD); (6) for multiple similar studies from the same research institution, a recent or higher quality study will be selected.

\section{Exclusion criteria}

(1) The literature including cases of open surgery or hand-assisted LTG; (2) the literature that did not, respectively, provide the data for linear stapler group and circular stapler group or the surgical method was not clearly stated in the literature; (3) the literature was a case report, case series, letters, review or non-control study without control group; (4) the sample size was too small, and the number of cases was less than 20 cases. The studies with fewer than 20 cases are usually considered small-sample studies and were excluded by authors in some published meta-analysis articles. $^{18}$ (5) Other treatments were differently 


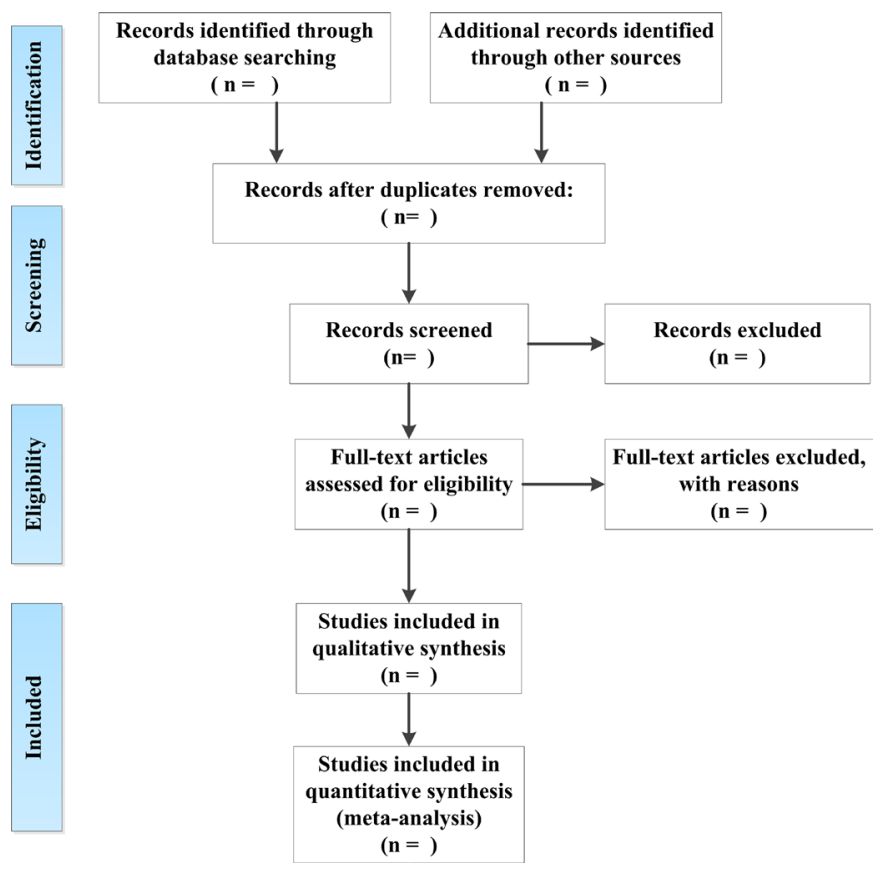

Figure 1 Flow diagram of study selection.

performed between two groups during preoperation and postoperation, and these treatments probably affected the observed outcome in the studies; (6) the literature was a repeated publication.

\section{Study screening and selection}

Any duplication will be identified and removed using the EndNote X8 reference management software (Clarivate Analytics, Thomson Place, Boston, USA). Under the pre-established inclusion and exclusion criteria, the titles and abstracts of all remaining literatures are carefully read and examined to exclude obviously unrelated documents. The full text of the screened literature will be deeply and carefully read to determine whether it is to be included. All steps will be independently conducted and cross-checked by three reviewers, and all disagreements will be resolved by discussion with the senior authors (Xueqing Yao) until a consensus be reached. The detailed process of study selection will be displayed in detail in a PRISMA-compliant flow diagram (figure 1).

\section{Data extraction and outcomes of interest}

Three reviewers will independently extract the data, and any discrepancy will be resolved by discussion until a consensus is reached. All extracted data will be filled in data extraction sheets created by Microsoft Excel 2010 (Microsoft Corporation, Redmond, Washington, USA). The main extracted information are as follows: (1) study characteristics (eg, first author's name, year of publication, country of study, study design, study period, number of patients, number of patients with linear stapler, number of patients with circular stapler); (2) participant characteristics (eg, age, sex, ethnicity, body mass index (BMI), cancer stage, American Society of Anesthesiologists (ASA) score); (3) primary outcomes: anastomotic leakage, anastomotic stricture, anastomotic haemorrhage, total postoperative complications; (4) secondary outcomes: time to first instance of passing gas after surgery, first feeding time, total operation time, reconstruction time of digestive tract, estimated blood loss, lymph node harvest, the distance from the proximal margin of the tumour, postoperative hospital stay. Any missing information is supplemented by contacting the original author by telephone or email.

\section{Quality assessment}

The quality of the studies will be independently scored by three reviewers using the Cochrane risk of bias tool or the Newcastle-Ottawa Quality Assessment Scale (NOS) ${ }^{19}$ The methodological quality of RCTs will be assessed using the Cochrane risk of bias tool. ${ }^{20}$ The methodological quality of non-random studies such as case-control and cohort studies will be assessed by the NOS, which consists of three factors: patient selection, comparability of the study groups and assessment of outcome. A score of 0-9 (allocated as stars) will be allocated to each study except for RCTs. RCTs and observational studies achieving six or more stars will be considered to be of high-quality studies. In cases where discrepancies arose, studies will be re-examined and a consensus will be reached through discussion.

\section{Statistical analysis}

All the meta-analyses will be performed using Review Manager V.5.3 (Cochrane Collaboration, Oxford, UK). The WMD and OR will be used to compare continuous and dichotomous variables, respectively, and all the results will be reported with $95 \%$ CIs. For the literature reporting median and range of continuous variables, the mean and SD will be extracted using the method described by Hozo $e t a l .^{21}$ Continuous variables that only provided quartiles or mean and SD could not be extracted will be eliminated. Assessment of statistical heterogeneity among the studies will be undertaken using the $\chi^{2}$ and $\mathrm{I}^{2}$ statistical tests. Where there is no obvious statistical heterogeneity among the studies as denoted by a $p$ value $\geq 0.1$ or $\mathrm{I}^{2} \leq 50 \%$, the fixed effect model will be used for meta-analysis. Conversely, in cases where statistical heterogeneity is observed among studies with a $\mathrm{p}$ value $<0.1$ or $\mathrm{I}^{2}>50 \%$, a random effect model will be used for meta-analysis. If concerns for high heterogeneity $\left(\mathrm{I}^{2}\right.$ value $>75 \%$ indicates high heterogeneity $)^{22}$ exist, a sensitivity analysis will be performed.

\section{Assessment of publication bias}

The potential publication bias will be investigated using funnel plots drawn by the STATA SE V.12.0 software. The publication bias will be assessed by visual inspection of the Begg's funnel plots, whereby, if the SE of logOR of each study is plotted against its logOR, an asymmetric plot suggests a possible publication bias. ${ }^{23}$ In addition, the asymmetry of the funnel-plot will be assessed using 
the Egger linear regression test at the $\mathrm{p}<0.10$ significance level. $^{24}$

\section{Subgroups analysis}

To explore the potential heterogeneity, subgroup meta-analyses will be performed based on different characteristics of the patient (eg, age, sex, ethnicity, BMI, cancer stage) as well as by study characteristics (eg,country of study, study design, year of publication, study period, number of patients).

\section{Sensitivity analysis}

In order to ensure the robustness and reliability of evidence, sensitivity analysis will be performed to assess the effect of studies with a high risk of bias. The results will be compared to decide whether low-quality studies should be excluded based on sample size and quality assessment of studies or effect on pooled effective size. In addition, a leave-one-out sensitivity meta-analysis might be considered if a study involving a large number of patients was based on different types of studies. ${ }^{25}$

\section{Patient and public involvement}

Not applicable. Patient and public involvement will not be required because this proposed systematic review and meta-analysis is based on previously published data, which does not include intervention data on patients.

\section{Ethics and dissemination}

Ethical approval will not be required because this proposed systematic review and meta-analysis is based on previously published data, which does not include intervention data on patients. The findings of this study will be submitted to a peer-reviewed journal and will be presented at a relevant congress.

\section{DISCUSSION}

Recent decades have witnessed significant advancements in the skills set and the equipment for laparoscopic surgery advance. This has not only expanded the application scope of laparoscopic surgery in gastric cancer, ${ }^{26}{ }^{27}$ but has also lead to the transition of laparoscopic reconstruction of the digestive tract in gastric cancer from laparoscopic-assisted surgery to totally laparoscopic surgery. ${ }^{6}$ However, the application of TLTG for total laparoscopic digestive tract reconstruction faces some difficulties due to its high technical requirements. ${ }^{1428}$ However, total laparoscopic digestive tract reconstruction after TLTG has obvious theoretical advantages. ${ }^{29}{ }^{30}$ For instance, the pneumoperitoneum provides a larger operation space for surgery and the multiangle lens provides direct vision for operation to avoid damage. Therefore, TLTG is a promising technique for gastric cancer.

It is no doubt that the development of stapler technology has promoted the development of laparoscopic gastrointestinal operation, especially in TLTG. Presently, mechanical anastomosis for oesophagojejunostomy in TLTG is mainly divided into two types: end-to-side anastomosis using the circular stapler and side-to-side/functional end-to-end anastomosis using the linear stapler. ${ }^{6-8}$

The circular stapling anastomosis method is divided into different methods according to the placement of the nail anvil: the traditional method of direct insertion, reverse anvil method and OrVil method. ${ }^{31-33}$ However, in the first two methods, the main body of the stapler cannot enter the abdominal cavity through the trocar, which requires that the pneumoperitoneum be closed and a small auxiliary incision is often created, thereby reducing the fluency and efficiency of the operation. In addition, the difficult in operation of the oesophageal purse suture and the placement of the nail anvil also limits the application of these two methods. Although the OrVil method does not require the placement of an anvil through the abdominal cavity, it requires the services of an anaesthesiologist and a special anvil placement device. ${ }^{9}$ The price of the special device is high, and the extraction of the guide tube might cause intra-abdominal infection. ${ }^{928} 34$

Linear stapling anastomosis involves functional end-to-end anastomosis (FEEA method) as well as side-toside anastomosis (Overlap method). ${ }^{35}$ This technique is appropriate for total laparoscopic gastrectomy compared with using circular stapling anastomosis. ${ }^{11} 3034$ Based on the published literatures and the experience of our centre, the linear stapler has the following advantages ${ }^{29} 3637$ : (1) linear stapler can be easily put into the abdominal cavity via a trocar and has a better visual field; (2) the operation of linear stapler is simple and convenient, and the requirement for the surgeon is lower compared with using a circular stapler; (3) the circular stapler with two rows of staples, but the linear stapler provides three rows of nail technology to theoretically improve the safety of the anastomosis. However, although some advantages have been reported for linear stapler, its application in LTG has some limitations such as ${ }^{814}$ (1) a longer stump of the oesophagus is required which limits the incisal margin; (2) when the anastomosis plane is higher than the plane of oesophageal hiatus, the operation is performed in a narrow thoracic cavity and the visual field is narrowed; (3) the pulling and folding of the jejunum arm might increase the tension in the anastomosis. Whether the possibly increased tension could increase the risk of anastomotic leak is an important topic needed to be resolved in this study. The discussed anastomotic methods have their advantages and disadvantages in the anastomosis of the oesophagus between jejunum, and it is not clear which anastomosis technique is superior. ${ }^{14}$ Further, no standard methods have been established to guide the selection. ${ }^{3839}$ Therefore, it is meaningful and necessary to conduct a systematic review and meta-analysis to provide a reference that could aid clinical surgeons in choosing a more appropriate alternative for their patients.

In this review, in order to collect all existing and available literature, RCTs and non-RCTs as well as observational studies will be included. Because of the novelty of this research topic, a few studies had been reported. However, the non-RCTs and observational studies might 
affect the quality of the evidence and lower the confidence level of the result. Besides, there are many factors such as different standards of choosing patients, different proficiency in laparoscopic techniques and different habits or methods of using the stapler by different surgeons in different regions, which might influence the results. Hence, in view of these, it is very important for this review to perform subgroup analysis and sensitivity analysis. Further analysis and explanations will be carried out in our review to ensure the robustness and reliability of the results.

In summary, this systematic review and meta-analysis will help to determine the differences in terms of safety and efficacy between linear stapler and circular stapler in TLTG. Furthermore, the findings of this study will not only help the surgeons in choosing the surgical methods, but also might benefit more patients in the future.

\section{Author affiliations}

${ }^{1}$ Gastrointestinal Surgery, Shunde Hospital, Southern Medical University (The First People's Hospital of Shunde Foshan), Foshan, China

${ }^{2}$ Graduate School, Guangdong Medical University, Zhanjiang, China

${ }^{3}$ Department of General Surgery, Guangdong Provincial People's Hospital

(Guangdong Academy of Medical Sciences), Guangzhou, China

${ }^{4}$ The Second School of Clinical Medicine, Southern Medical University, Guangzhou, China

Contributors Wrote the paper: TL, LD. Study concept and design: M0, XY. Registered the protocol in the PROSPERO database: TL, MO. Preliminary literature search: TL, LD. Corrected and revised manuscript: MO, XY. Approving current version of manuscript: TL, LD, XY, MO.

Funding This study was financially supported by the Science and Technology Planning Project of Guangdong Province (No. 2014A020212053, No.

2014A020212636, No. 2016A020215128, No. 2017A030223006), the Medical Scientific Research Fund Project of the Guangdong Province (No. B2013374, No. A2014718, No. A2017160), the Research Startup Project of Shunde Hospital of Southern Medical University (No. SRSP2018001), the Science and Technology Bureau of Foshan City (No. 201308247), and the 13th Five-Year Key Specialty Project of Foshan City (FSGSPZD135051).

Competing interests None declared.

Patient consent for publication Not required.

Provenance and peer review Not commissioned; externally peer reviewed.

Open access This is an open access article distributed in accordance with the Creative Commons Attribution Non Commercial (CC BY-NC 4.0) license, which permits others to distribute, remix, adapt, build upon this work non-commercially, and license their derivative works on different terms, provided the original work is properly cited, appropriate credit is given, any changes made indicated, and the use is non-commercial. See: http://creativecommons.org/licenses/by-nc/4.0/.

\section{REFERENCES}

1. Ferlay J, Soerjomataram I, Dikshit R, et al. Cancer incidence and mortality worldwide: sources, methods and major patterns in GLOBOCAN 2012. Int J Cancer 2015;136:E359-86.

2. Bertuccio $P$, Chatenoud L, Levi F, et al. Recent patterns in gastric cancer: a global overview. Int J Cancer 2009;125:666-73.

3. Lin $\mathrm{M}$, Huang $\mathrm{CM}$, Zheng $\mathrm{CH}$, et al. Totally laparoscopic total gastrectomy for locally advanced middle-upper-third gastric cancer. $J$ Vis Surg 2017;3:46.

4. Umemura A, Koeda K, Sasaki A, et al. Totally laparoscopic total gastrectomy for gastric cancer: literature review and comparison of the procedure of esophagojejunostomy. Asian J Surg 2015;38:102-12.

5. Kodera Y, Yoshida K, Kumamaru H, et al. Introducing laparoscopic total gastrectomy for gastric cancer in general practice: a retrospective cohort study based on a nationwide registry database in Japan. Gastric Cancer 2019;22:202-13.

6. Shim JH, Yoo HM, Oh SI, et al. Various types of intracorporeal esophagojejunostomy after laparoscopic total gastrectomy for gastric cancer. Gastric Cancer 2013;16:420-7.

7. Kawamura H, Ohno Y, Ichikawa N, et al. Anastomotic complications after laparoscopic total gastrectomy with esophagojejunostomy constructed by circular stapler (OrVil ${ }^{\mathrm{TM}}$ ) versus linear stapler (overlap method). Surg Endosc 2017;31:5175-82.

8. Yoshikawa K, Shimada M, Higashijima J, et al. Usefulness of the transoral anvil delivery system for Esophagojejunostomy after laparoscopic total gastrectomy: a single-institution comparative study of transoral anvil delivery system and the overlap method. Surg Laparosc Endosc Percutan Tech 2018;28:e40-3.

9. Jeong O, Park YK. Intracorporeal circular stapling esophagojejunostomy using the transorally inserted anvil (OrVil) after laparoscopic total gastrectomy. Surg Endosc 2009;23:2624-30.

10. Zhang N, Su X, Xu K. [Comparison of the application between circular stapler and linear stapler in Billroth II(anastomosis of distal gastrectomy]. Zhonghua Wei Chang Wai Ke Za Zhi 2018;21:201-5.

11. Son SY, Cui LH, Shin HJ, et al. Modified overlap method using knotless barbed sutures (MOBS) for intracorporeal esophagojejunostomy after totally laparoscopic gastrectomy. Surg Endosc 2017;31:2697-704.

12. Amisaki M, Kihara $\mathrm{K}$, Endo $\mathrm{K}$, et al. Comparison of singlestapling and hemi-double-stapling methods for intracorporeal esophagojejunostomy using a circular stapler after totally laparoscopic total gastrectomy. Surg Endosc 2016;30:2994-3000.

13. Ding W, Tan Y, Xue W, et al. Comparison of the short-term outcomes between delta-shaped anastomosis and conventional Billroth I anastomosis after laparoscopic distal gastrectomy: a meta-analysis. Medicine 2018;97:e0063.

14. Sugiyama M, Oki E, Ogaki K, et al. Clinical outcomes of esophagojejunostomy in totally laparoscopic total gastrectomy: a multicenter study. Surg Laparosc Endosc Percutan Tech 2017;27:e87-91.

15. Moher D, Shamseer L, Clarke M, et al. Preferred reporting items for systematic review and meta-analysis protocols (PRISMA-P) 2015 statement. Syst Rev 2015;4:1.

16. Moher D, Liberati A, Tetzlaff J, et al. Preferred reporting items for systematic reviews and meta-analyses: the PRISMA statement. Int $J$ Surg 2010;8:336-41.

17. Booth A, Clarke M, Dooley G, et al. The nuts and bolts of PROSPERO: an international prospective register of systematic reviews. Syst Rev 2012;1:2.

18. Xinming S, Zhihui C, Shicai C, et al. Lateral lymph node dissection for rectal cancer: a meta analysis. Journal of Colorectal \& Anal Surgery 2009;15:217-20.

19. Wells GA, Shea B, O'Connell D, et al. The Newcastle-Ottawa Scale (NOS) for assessing the quality of nonrandomised studies in metaanalyses. Ottawa, Ontario: The Ottawa Health Research Institute, 2011.

20. Higgins JP, Altman DG, Gøtzsche PC, et al. The Cochrane Collaboration's tool for assessing risk of bias in randomised trials. BMJ 2011;343:d5928.

21. Hozo SP, Djulbegovic B, Hozo I. Estimating the mean and variance from the median, range, and the size of a sample. BMC Med Res Methodol 2005;5:13

22. Higgins JP, Thompson SG, Deeks JJ, et al. Measuring inconsistency in meta-analyses. BMJ 2003;327:557-60.

23. Stuck AE, Rubenstein LZ, Wieland D. Bias in meta-analysis detected by a simple, graphical test. Asymmetry detected in funnel plot was probably due to true heterogeneity. BMJ 1998;316:469.

24. Egger M, Davey Smith G, Schneider M, et al. Bias in meta-analysis detected by a simple, graphical test. BMJ 1997;315:629-34.

25. Wang ZY, Zhao K, Zheng J, et al. Systematic review and metaanalysis of the association between complement factor $\mathrm{H} \mathrm{I62V}$ polymorphism and risk of polypoidal choroidal vasculopathy in Asian populations. PLoS One 2014;9:e88324.

26. Omori T, Oyama T, Mizutani S, et al. A simple and safe technique for esophagojejunostomy using the hemidouble stapling technique in laparoscopy-assisted total gastrectomy. Am J Surg 2009;197:e13-17.

27. Kim HH, Hyung WJ, Cho GS, et al. Morbidity and mortality of laparoscopic gastrectomy versus open gastrectomy for gastric cancer: an interim report--a phase III multicenter, prospective, randomized Trial (KLASS Trial). Ann Surg 2010;251:417-20.

28. Cianchi F, Macrì G, Indennitate G, et al. Laparoscopic total gastrectomy using the transorally inserted anvil (OrVil ${ }^{\mathrm{TM}}$ ): a preliminary, single institution experience. Springerplus 2014;3:434. 
29. Kim EY, Choi HJ, Cho JB, et al. Totally laparoscopic total gastrectomy versus laparoscopically assisted total gastrectomy for gastric cancer. Anticancer Res 2016;36:1999-2003.

30. Umemura A, Koeda K, Fujiwara $\mathrm{H}$, et al. Totally Laparoscopic Total Gastrectomy for Gastric Cancer at a Single Institutional Experience and Changes in Technique of Esophagojejunostomy. Indian J Surg 2016;78:249-53.

31. Okabe H, Tsunoda S, Tanaka E, et al. Is laparoscopic total gastrectomy a safe operation? A review of various anastomotic techniques and their outcomes. Surg Today 2015;45:549-58.

32. Ito $\mathrm{H}$, Inoue $\mathrm{H}$, Odaka $\mathrm{N}$, et al. Evaluation of the safety and efficacy of esophagojejunostomy after totally laparoscopic total gastrectomy using a trans-orally inserted anvil: a single-center comparative study. Surg Endosc 2014;28:1929-35.

33. Lacerda CF, Bertulucci PA, Oliveira AT. Step-by-step esophagojejunal anastomosis after intra-corporeal total gastrectomy for laparoscopic gastric cancer treatment: technique of "reverse anvil". Arq Bras Cir Dig 2014;27:71-6.

34. Li X, Ke C. [Recent advances of anastomosis techniques of esophagojejunostomy after laparoscopic totally gastrectomy in gastric tumor]. Zhonghua Wei Chang Wai Ke Za Zhi 2015;18:512-5.
35. Miura S, Kanaya S, Hosogi H, et al. Esophagojejunostomy with linear staplers in laparoscopic total gastrectomy: experience with 168 cases in 5 consecutive years. Surg Laparosc Endosc Percutan Tech 2017;27:e101-e07.

36. Lee IS, Kim TH, Kim KC, et al. Modified techniques and early outcomes of totally laparoscopic total gastrectomy with side-toside esophagojejunostomy. J Laparoendosc Adv Surg Tech A 2012;22:876-80.

37. Gong CS, Kim BS, Kim HS. Comparison of totally laparoscopic total gastrectomy using an endoscopic linear stapler with laparoscopicassisted total gastrectomy using a circular stapler in patients with gastric cancer: A single-center experience. World J Gastroenterol 2017;23:8553-61.

38. Bracale U, Marzano E, Nastro P, et al. Side-to-side esophagojejunostomy during totally laparoscopic total gastrectomy for malignant disease: a multicenter study. Surg Endosc 2010;24:2475-9.

39. Hirahara N, Tanaka T, Yano S, et al. Reconstruction of the gastrointestinal tract by hemi-double stapling method for the esophagus and jejunum using EEA OrVil in laparoscopic total gastrectomy and proximal gastrectomy. Surg Laparosc Endosc Percutan Tech 2011;21:e11-15. 